\section{Extra extra}

Many types of leukaemia have been associated with chromosomal translocations that form oncogenic fusion proteins. In the October issue of Nature Genetics, Anne Hagemeijer and colleagues describe a unique fusion between NUP214 and ABL1 that occurs only on episomes in human T-cell acute lymphoblastic leukaemia (T-ALL) cells. This genetic aberration could not be detected by conventional cytogenetics and is the first episomal fusion gene associated with human cancer.

In studying the role of the tyrosine kinase ABL1 in T-ALL, the authors observed that $A B L 1$ was amplified in about $6 \%$ of patients with this cancer. Interestingly, these gene amplifications were all extra-chromosomal. Further analysis of the ABL1-carrying episome revealed that it contained several other genes, including NUP214, and that all these genes were derived from the chromosome region $9 q 34$. The authors discovered that this chromosomal region becomes amplified and circularized, leading to formation of an episome that carried an in-frame fusion between the carboxyl terminus of ABL1 and the amino terminus of NUP214. Examination of leukaemic cells from 175 patients with T-ALL revealed that 10 (6\%) expressed NUP214-ABL1.

How might this fusion protein contribute to cell transformation? First, it contains the coiled-coil oligomerization motif of NUP214, which mediates the protein-protein interactions of this nuclear-pore protein. Second, it contains the $\mathrm{SH} 3, \mathrm{SH} 2$ and kinase domains of ABL1, allowing it to function as a constitutively active tyrosine kinase. Hagemeijer and colleagues found that the fusion protein was also expressed by three T-ALL cell lines, and that one of the known substrates of ABL1, CRKL, was constitutively phosphorylated in these cells. Importantly, treatment of these cells with imatinib, a selective inhibitor of ABL1 kinase activity, decreased CRKL phosphorylation in all three cell lines and inhibited the proliferation of one cell line. Phosphorylation of CRKL was also inhibited by imatinib in primary bone-marrow cells isolated from one of the patients found to carry the fusion gene.

Additional leukaemia-associated mutations were also detected in bonemarrow cells of these patients. These mutations were included in the authors' multistep model of T-ALL pathogenesis, beginning with deletion of tumour suppressors (CDKN2A and $C D K N 2 B$ ), overexpression of a transcription factor (HOX11) and, finally, expression of the constitutively active tyrosine kinase NUP214-ABL1. As expression of the NUP214-ABL1 fusion protein was associated with an especially aggressive course of disease, imatinib will be an important new therapeutic approach for these patients. The authors also emphasize that genome-analysis tools such as high-resolution array comparative genome hybridization are important for detecting cancer-associated genetic amplifications and deletions that cannot be detected by conventional cytogenetics.

Kristine Novak

\section{(2) References and links} ORIGINAL RESEARCH PAPER Grau, C. et al. Fusion of NUP214 to ABL1 on amplified episomes in T-cell acute lymphoblastic leukemia. Nature Genet. 12 Sept 2004 (doi:10.1038/ng1425)

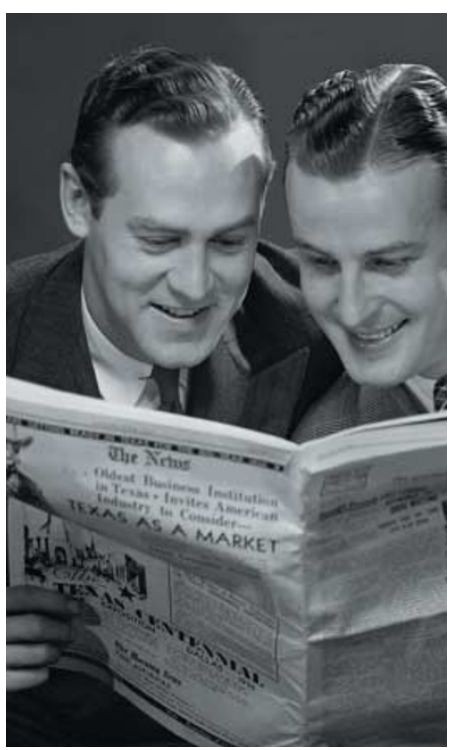

TRIAL WATCH

\section{Predictions are circulating}

We know that tumour cells disseminate round the body through the blood to form metastases, but measurement of circulating tumour cells has been difficult until recently. Massimo Cristofanilli, Daniel Hayes and colleagues have used a new technique to detect tumour cells in whole blood to show that circulating tumour cells in patients with metastatic breast cancer are associated with short survival.

The technique called CellSearch (developed by Veridex) enriches a blood sample for epithelial cells using magnetic beads coated with antibodies against epithelial cell-adhesion molecules. Then, fluorescently labelled antibodies against epithelial-lineage-associated cytokeratins and the leukocyte antigen CD45, together with a nuclear stain, further separate out the epithelial cells. The nucleated, CD45-negative, cytokeratin-positive cells are then counted.

The prospective multicentre study included 177 patients with metastatic breast cancer who had had previous adjuvant treatment and/or treatment for metastatic disease. The levels of circulating tumour cells were measured before patients started a new treatment, and then again at the first follow-up visit 3-4 weeks later. The control group consisted of 72 premenopausal healthy women, 73 postmenopausal healthy women, 99 women with benign breast disease and 101 women with non-malignant diseases.

The first 102 patients with metastatic breast cancer enrolled were used as the training set to select the level of circulating tumour cells to be used to stratify patients into favourable and unfavourable prognosis. Circulating epithelial cells were rare in the control group (less than 2 cells per $7.5 \mathrm{ml}$ blood), but $61 \%$ of patients with metastatic breast cancer had 2 or more cells per $7.5 \mathrm{ml}$ blood. The levels measured in the training set were correlated with progression-free survival (PFS) and overall survival (OS), and a cut-off point of 5 cells per $7.5 \mathrm{ml}$ blood was chosen to distinguish unfavourable and favourable prognosis. When the next 75 patients who were enrolled - the validation set - were evaluated, the ability of the cut-off point chosen to predict outcome was confirmed.

For the 87 patients with more than 5 cells per $7.5 \mathrm{ml}$ of blood, the median PFS was 2.7 months and median OS was 10.1 months - significantly shorter than the PFS of 7 months and OS of over 18 months seen in the 90 patients with less than 5 cells per $7.5 \mathrm{ml}$ of blood. Ten patients who died before the follow-up visit all had high to extremely high levels of circulating tumour cells.

Hormone-receptor status was significantly associated only with OS, but, although time to metastasis, ERBB2 status and type of therapy remained relevant in the multivariate analysis, circulating tumour cells emerged as the strongest predictor of PFS and OS. The association with PFS and OS was not robust in the patients receiving hormonal therapy or immunotherapy.

So, the test reliably estimated disease prognosis and survival much earlier (3-4 weeks) than in usual clinical practice with traditional imaging methods, which are usually done 8-12 weeks after initiation of treatment. Comparison of the CellSearch assay with measurement of serum tumour markers, such as CA15-3, and further investigation of the robustness of the test for prediction of outcome for different types of therapy would be useful next steps.

ORIGINAL RESEARCH PAPER Cristofanilli, M. et al. Circulating tumor cells, disease progression, and survival in metastatic breast cancer. N. Engl. J. Med. 351, 781-791 (2004) 